\title{
Design of Unmanned Surface Vessels Experiment Platform
}

\author{
Changshun WANG \\ School of Information Science and Electric Engineering, Shandong Jiaotong University, Jinan, \\ 250357, China \\ Email: shun841030@163.com
}

Key words: Unmanned Surface Vessels, USV, Experiment Platform, Course Tracking

\begin{abstract}
In recent years, More and more attention has been paid to Unmanned Surface Vessels motion control field. An experimental platform for USV based on wireless communication was introduced for USV motion control algorithm experiments in this paper. The structure and implementation of experimental platform was described in detail. Stability and reliability tests for experimental platform were carried out by following an circular path, and the USV state data in sailing was analyzed by drawing tools in MATLAB. Experimental results show that the platform can complete algorithm validation experiments accurately.
\end{abstract}

\section{Introduction}

In recent years, Unmanned Surface Vessels(USV) has played an important role in search and rescue, marine environmental exploration, oceanographic research and marine rights due to its advantages such as flexibility, concealment, can be carried in other ships. It has been developed as an important direction of military equipment in the United States, Israel and lots of other countries [1]. Path following control and formation control as in paper [2] and [3] has been an hot topics in order to facilitate large-scale autonomous operations. The computer simulation technology such as MATLAB has laid a good theoretical basis in order to guarantee the algorithm reliability and stability. But traditional dynamic and kinematic model calculation methods and fluid dynamics computational software were usually used independently due to the complex marine environment. They can't replace each other functionally. Thus, the simulation can't ensure that the algorithm could be applied to the USV directly. It was often difficult to achieve real ship tests because of financial constraints. An novel experimental platform was designed in this paper which included PC and surface experiment equipment. The surface equipment was controlled by the PC which run the control algorithm and received the surface equipment state information on shore. It achieved very good results in the actual test.

\section{Structure of Platform}

The structure of experimental platform was shown in figure 1, which was divided into shore platform and USV experimental platform. The shore platform consists of a computer and a long-distance wireless data transmission module. Control algorithm was executed in the computer, but all the control information was sent to the USV experimental platform through the wireless data transmission module. USV experimental platform was a miniature surface vessel equipped with a wireless communication module, Beidou modules, electronic monitoring module course and two propulsion propellers can sail in the water. It was capable of receiving control commands issued by 
the shore platform, while its location, speed, heading, propeller speed, battery charge and other information was sent back to shore platform.

USV structure was shown in figure 1, the Port and Starboard propellers could produce the thrust power when the motors rotate. When the speed is different, the thrust is imbalance, and so the USV course will change continuously. When the USV sailing at full speed, we can control the pitch angle through the control device at stern. USV location and attitude information got from GPS and AHRS will be sent to the control terminal through wireless communication module. Also the USV controller can get navigation target information from the control terminal in the same way.

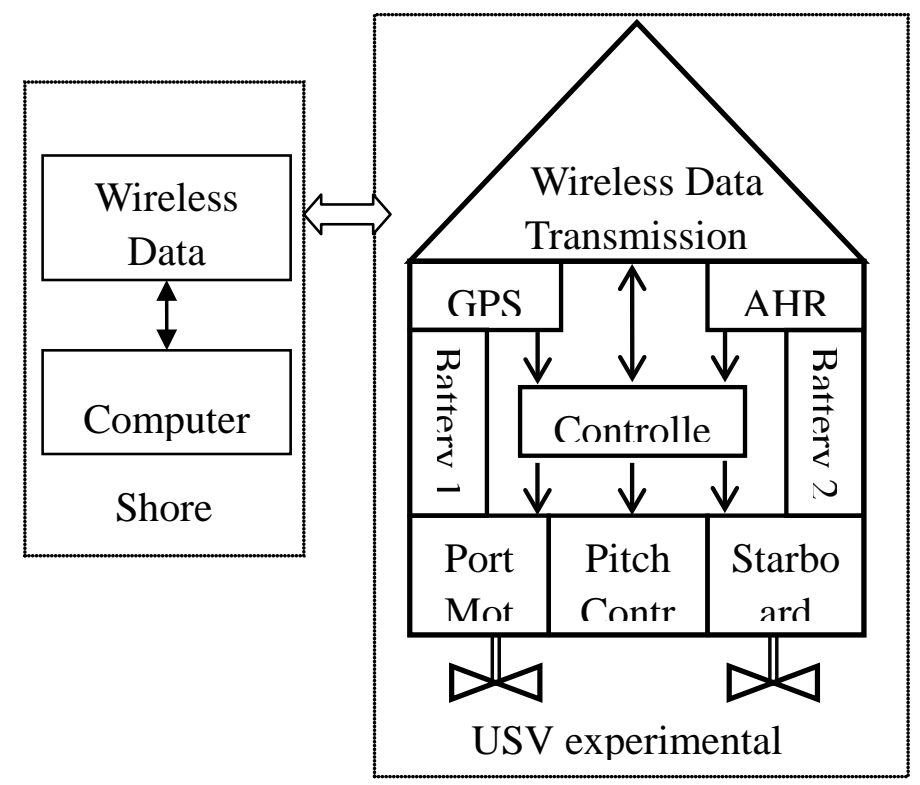

Fig. 1 Structure of the Experiment Platform

\section{Design Of The USV Experiment Platform}

Selection Of The USV. Since the USV characteristics of high sailing speed and flexibility, the USV platform chosen in this paper had the parameters: Long $0.11 \mathrm{~m}$, Width $0.36 \mathrm{~m}$, a maximum speed of 15 knots, weighs $15 \mathrm{Kg}$ with battery, the minimum radius of gyration $6.4 \mathrm{~m}$. Both sides had a propulsion motor of $36 \mathrm{~W}$ rated power and a set of interchangeable $50 \mathrm{AH}$ battery. Normally the 1st battery power to the control circuit, the 2nd battery to the propulsion motor. In case of a serious shortage of electricity during testing, the powered battery can be remotely exchanged by the shore platform to ensure safe return.

Design of control system. Considering the amount of data transmitted and the reliability of wireless communication, the communication period was designed to $1 \mathrm{~S}$. Therefore, the controller needs to complete the tasks such as control commands receiving, USV status data collection and sending, the USV control within 1S. ST's Cortex-M3 core ARM controller STM32F103VBT6 was chosen as the core controller. And WSN-03 was chosen as the wireless data transmission module with the parameters as follows: half-duplex communication, the maximum transmission distance of 4Km, TTL serial port. The control system circuit schematic was shown in Figure 2. 


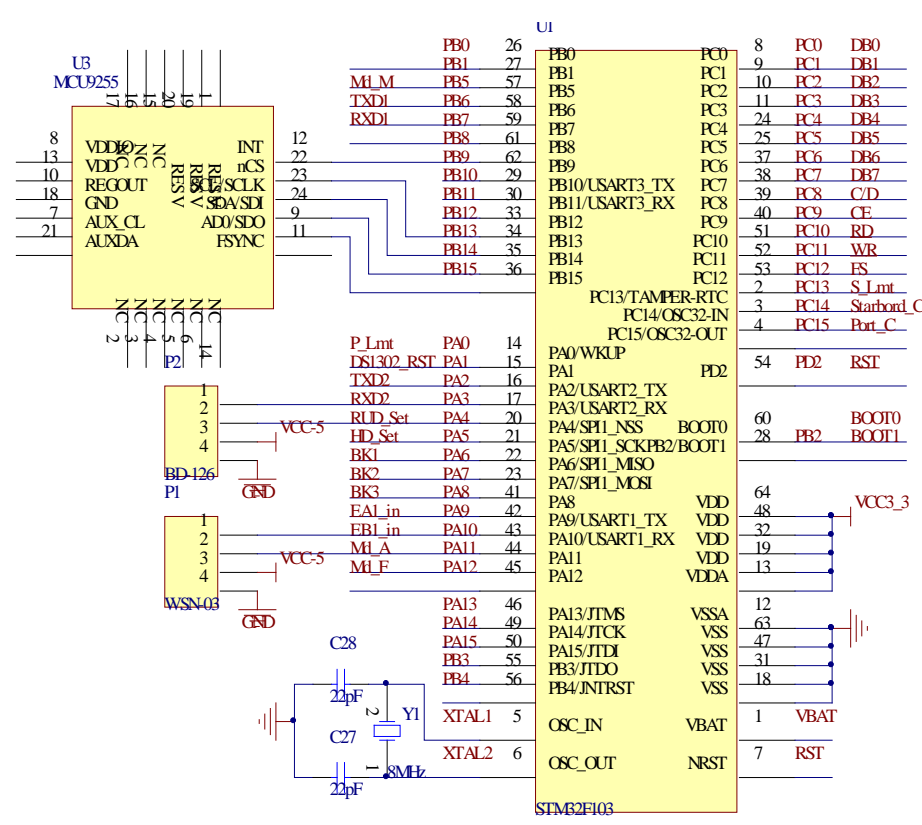

Fig. 2 MCU schematic

USV location and velocity was provided by the Beidou positioning module, therefore, the control accuracy could be impacted by the accuracy of positioning module. BD-126 Compass / GPS dual-mode positioning module was chosen for USV positioning, as shown in figure 2. The positioning and velocity information would be sent through its GGA and VTG sentence which was organized in NMEA0183 format [5].

Design Of AHRS.As shown in Figure 3, AHRS designed in the paper consist of a 3-axis accelerometer, a 3-axis gyroscope, a 3-axis magnetometer sensor and a ARM processer STM32F107 [4]. All the sensors were integrated into a single chip MPU9255, a 9-axis Motion Tracking device from InvenSense Company.

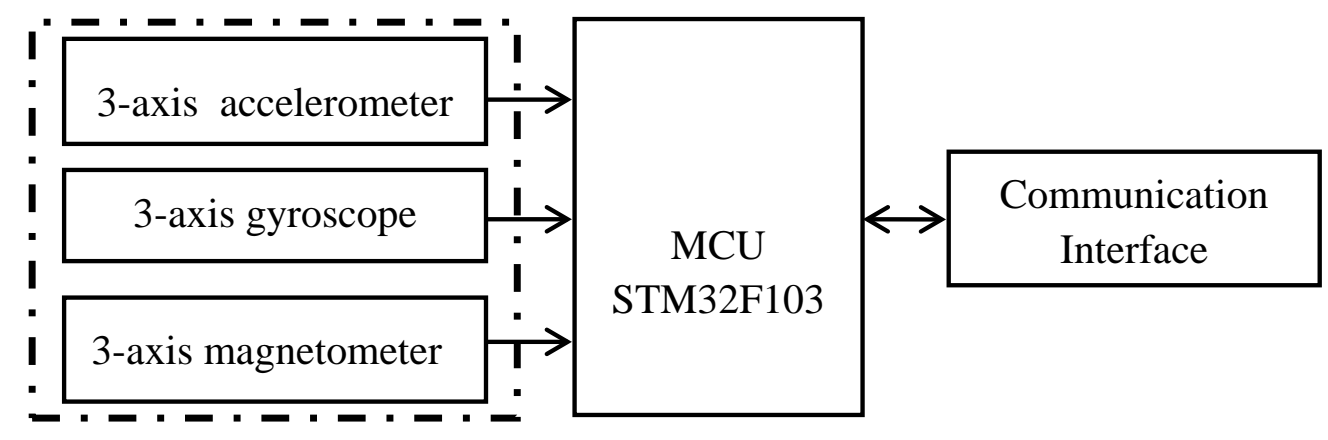

Fig. 3 Diagram of AHRS

The basic step of AHRS could be described as in paper[6, 7]:

(a)The three acceleration $\left(a_{x}, a_{y}, a_{z}\right)$ can be obtained from 3-axis accelerometer in static conditions. And then we can calculate the Roll angle $\varphi$ and Pitch angle $\theta$ with Euler formula as in Eq. (1): 


$$
\left\{\begin{array}{l}
\phi=\tan ^{-1}\left(\frac{a_{y}}{a_{z}}\right) \\
\theta=\tan ^{-1}\left(\frac{a_{x}}{\sqrt{a_{y}^{2}+a_{z}^{2}}}\right)
\end{array}\right.
$$

(b)Then we can get the compensated magnetic field strength $M_{n}=\left[\begin{array}{llll}m_{x} & m_{y} & m_{z}\end{array}\right]^{T}$ from 3-axis magnetometer's origin data $M_{b}=\left[\begin{array}{lll}m_{x}^{\prime} & m_{y}^{\prime} & m_{z}^{\prime}\end{array}\right]^{T}$ through the coordinate rotation and compensation as in Eq. (2).

$$
M_{b}=\left[\begin{array}{ccc}
\cos \theta & \sin \theta \sin \phi & \sin \theta \cos \phi \\
0 & \cos \phi & -\sin \phi \\
-\sin \theta & \cos \theta \sin \phi & \cos \theta \cos \phi
\end{array}\right]\left[\begin{array}{c}
m_{x} \\
m_{y} \\
m_{z}
\end{array}\right]
$$

(c)Thus, the compensated heading in static condition was computed as in Eq.(3):

$$
\psi=\tan ^{-1} \frac{m_{y}^{\prime}}{m_{x}^{\prime}}
$$

(d)Multi-sensor data fusion by quaternion, and obtain the final USV heading data using a Kalman filter algorithm.

USV Mathematical Model.USV mathematical model was need for control. The USV movement can be represented by the following equation [8].

(a) USV Plane motion equation

USV in calm water was powered by propeller propulsion and resistance. If the total weight of the USV was denoted as m, it can be obtained in accordance with Newton's second law:

$$
m \cdot \frac{d v}{d t}=F_{P}+F_{S}-f
$$

(b)USV rotation equation

When the propulsion from the two propeller was not equal to each other, the USV heading would change as Eq. (5).

$$
J \cdot \frac{d \omega}{d t}+k_{\omega} \cdot \omega=\frac{b}{2}\left(F_{s}-F_{P}\right)
$$

Where, J was the USV moment of inertia; $k_{\omega}$ was the rotational damping coefficient. USV heading change can be obtained by rotation angular velocity integration:

$$
\theta=\int_{0}^{t} \omega \cdot d t
$$

(c) propeller characteristic equation

According to the characteristics of the propeller, the propeller thrust generated can be obtained as follows:

$$
F_{i}=K_{F_{i}} \cdot \rho \cdot r_{i}^{2} \cdot D_{i}^{4}
$$


Where, $K_{F_{i}}$ was the propeller coefficient; $\rho$ for the density of water; $r_{i}$ for the propeller speed; $D_{i}$ for propeller diameter; $\mathrm{i}=\mathrm{P}$ or $\mathrm{S}$, denoted port and starboard propeller respectively.

Substituting Eq. (6) and (7) into (5), nonlinear USV model between propeller speed and heading would be described as follows:

$$
J \cdot \frac{d^{2} \theta}{d t}+k_{\omega} \cdot \frac{d \theta}{d t}=\frac{b}{2}\left(K_{F_{S}} \cdot \rho \cdot r_{S}^{2} \cdot D_{S}^{4}-K_{F_{P}} \cdot \rho \cdot r_{P}^{2} \cdot D_{P}^{4}\right)
$$

\section{Platform Test}

Test Method. Shown in Figure 4, it can be divided into four levels from the system function [9], that is,: guiding layer, control layer, actuator layer and physical layer. Sampling period of control algorithm in PC is equal to the communication cycle, namely 1S. But in USV experimental platform, the sampling period is much smaller than $1 \mathrm{~S}$.

Therefore, the guiding layer located on a computer would send guide instruction to USV platform and the control layer located in the USV platform would control the USV though the motors and propellers in actuator layer. Therefore, this test method is to send the speed and heading commands through the computer, and then monitor the USV platform status.

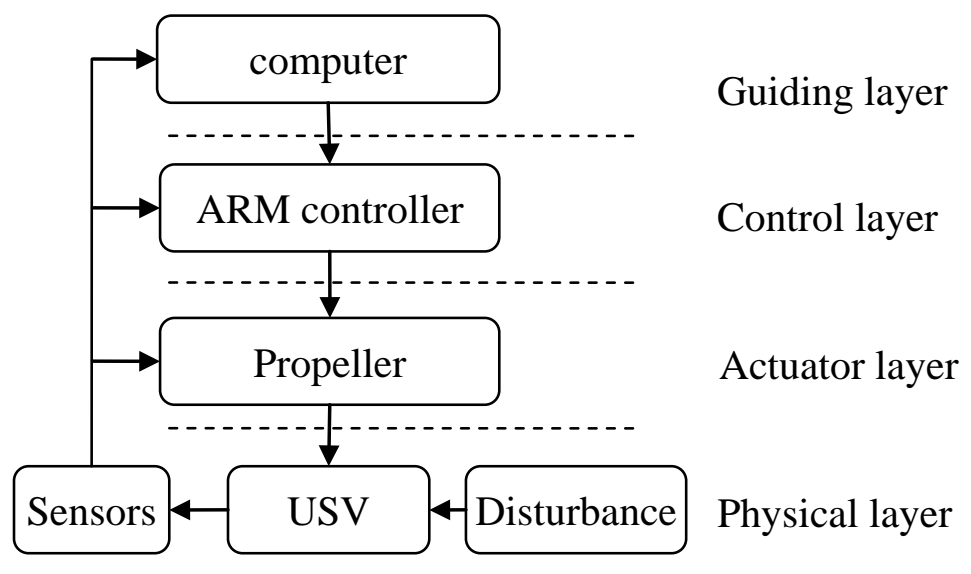

Fig. 4 Diagram of Experiment Platform

System Test Result. According to the above test methods, the experiment platform was tested in Jinan Changqing Lake under the conditions as wind speed $2 \mathrm{~m} / \mathrm{s}$, north, Beidou location: 116.811722E, 36.54253N. Given the USV velocity $2 \mathrm{~m} / \mathrm{s}$, a circular path with radius $20 \mathrm{~m}$, the test results are shown in Figure 5, the heading in the navigation shown in Figure 6.

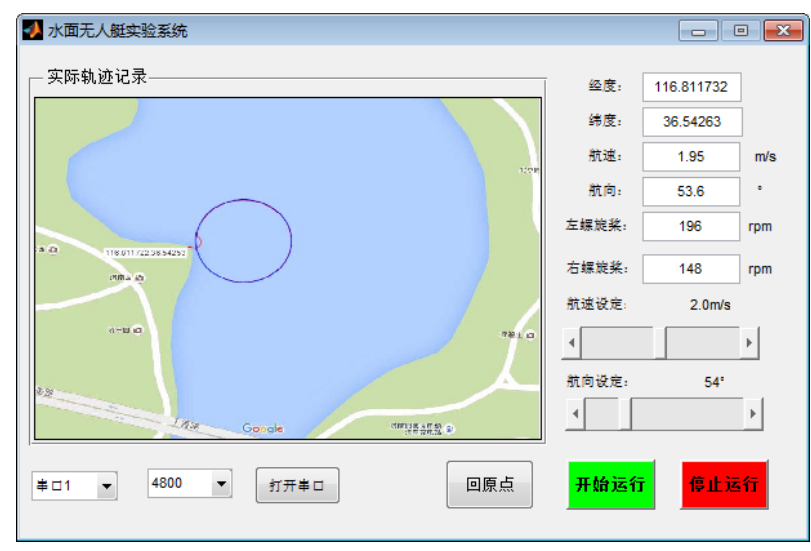

Fig. 5 System test result

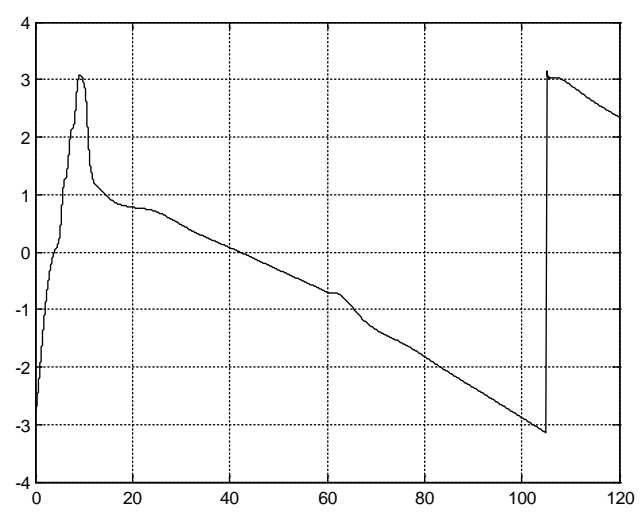

Fig. 6 Heading record in test 
As can be seen from the figure 5 and figure 6, the USV experimental platform can track circular path nicely, monitoring and data logging in computer is also fully in line with the true status of the USV experiment platform, indicating USV experimental platform well complete the navigation commands sent from the computer on shore, and achieve the USV control experiment.

\section{Conclusion}

After debugging, the USV experimental platform can achieve a control algorithm that it can be used in USV path following control, intelligent collision avoidance and formation control. After the lake test, the system has good stability and reliability.

\section{Acknowledgement}

This work is partially supported in part by Application Basic Research Projects of Ministry of Transport under Grant 2014329817130, and in part by A Project of Shandong Province Higher Educational Science and Technology Program under Grant J14LN35, and in part by Shandong Nature Science Foundation under Grants ZR2013ZEM006 and 2013ZRB01873, Innovation projects in Shandong Province Department of Transportation 2013A16-04.

\section{Reference}

[1] J LI. Development and Application of Unmanned Surface Vehicle[J]. Fire Control \& Command Control, Vol.37, NO.6, p.203-207, (2012).

[2] Y HU. Research on Tracking Control of Underactuated Unmanned Surface Vessels[D]. Harbin: Harbin Engineering University, (2011).

[3] L LIU, D WANG, Z PENG. Direct and composite iterative neural control for cooperative dynamic positioning of marine surface vessels[J]. NonlinearDyn, Vol.81, p.1315-1328, (2015).

[4] F.L. Markley. Attitude error representations for kalman filtering[J]. Journal of Guidance, Control, and Dynamics, Vol.63, No.2, p.311-317, (2003).

[5] D MA, C WANG, W PAN. Vehicle Fuel Monitoring Alarm System Based on Beidou Positioning System[J]. Microcontrollers \&Embedded Systems, No.1, p.60-63, (2015).

[6] P. Martin and E. Salaun. Design and implementation of a lowcost observer-based attitude and heading reference system. Control Engineering Practice, Vol.18, No.7, p.712-722, (2010).

[7] J.F. Guerrero-Castellanos, H. Madrigal-Sastre, S. Durand etc. Design and implementation of an Attitude and Heading Reference System (AHRS)[C], CCE 2011, (2011).

[8] X ZHANG, H FENG, Y XIONG etc. Nonlinear Modeling, Simulating and Experiment of an Unmanned Surface Vehicle Driven by Twin Electric Thrusters[J]. Ship Engineering, Vol.34, No.2, p.39-42,56, (2012).

[9] Y TIAN, D WANG, Z PENG. Design and validation of path tracking controller for USV along straight-lines[J]. Journal of Dalian Maritime University, Vol.41, No.4, p.14-18, (2015). 